\title{
A NEW METHOD FOR THE EXTRACTION OF MOSFET PARAMETERS AT AMBIENT AND LIQUID HELIUM TEMPERATURES
}

\author{
F. BALESTRA, I. HAFEZ and G. GHIBAUDO \\ Laboratoire de Physique des Composants à Semiconducteurs (CNRS-UA), \\ ENSERG/INPG, 23, AV. des Martyrs, F-38031 Grenoble Cedex, France
}

Résumê - Une méthode originale pour l'extraction des paramètres des Transistors MOS est présentée en fonction de la température. Cette méthode, qui repose sur une combinaison des caractéristiques de transfert donnant le courant de drain et la transconductance, permet d'obtenir des valeurs satisfaisantes pour les paramètres de tension de seuil et de mobilité.

Abstract - An original method for MOSFET parameter extraction is presented as a function of temperature. This method, which relies on combining drain current and transconductance transfer characteristics, enables reliable values of the threshold voltage and mobility parameters.

\section{1 - INTRODUCTION}

At ambient temperature, different methods for the extraction of the MOSFET parameters have been proposed [1-3]. These methods are based primarily on the premise that the threshold voltage $V_{t}$ can be obtained from extrapolating the linear region of the transfer characteristics $I_{d}\left(V_{g}\right)$ to zero drain current, or by taking the low field mobility $M_{0}$ equal to the maximum field effect mobility. At very low temperature, no reliable extraction method based on satisfactory mobility and current modelling has been proposed up til1 now. The aim of our method is to present a new parameter extraction which avoids the effect of mobility reduction with gate voltage on the determination of both the threshold voltage and low field mobility parameter. The extraction is conducted, as is usual, within the strong inversion regime of the MOSFET linear region and relies on the drain current $I_{d}$ and normalized transconductance $g_{m}\left(g_{m}=\left(1 / N_{d}\right) \cdot d I_{d} / d V_{g}\right)$ expressions $[4,5]$.

\section{2 - EXTRACTION METHOD AT AMBIENT TEMPERATURE}

a) Theory

The basic idea of our method consists of constructing a function which eliminates the influence of the mobility reduction with gate voltage ( At 300K the effective mobility is given by : Meff $\left.=\mu_{0} /\left[1+\theta\left(V_{\mathrm{g}}-V_{t}\right)\right]\right)$. This is achieved by the following function : 
$I_{d} /\left(g_{m}\right)^{1 / 2}=\left((W / L) C_{0 x} M_{0} V_{d}^{2}\right)^{1 / 2} \cdot\left(V_{g}-V_{t}\right)$

where $W$ and $L$ are the effective channel width and length respectively, $C_{0 x}$ is the gate oxide capacitance, $\theta$ is the mobility reduction coefficient, $\mu_{0}$ is the low field mobility, $V_{t}$ is the charge threshold voltage, $V_{g}$ and $V_{d}$ are the gate and drain voltages respectively.

Since this quantity $\left(I_{d} / g_{m}^{1 / 2}\right)$ is linear with the gate voltage, we can easily extract the real values of charge threshold voltage $\left(V_{t}\right)$ and low field mobility $\left(\mu_{0}\right)$ from the intercept and slope respectively. It is worth emphasizing that the present method al lows a separate determination of $V_{t}$ and $\mu_{0}$ which is not the case in the previous works [1-3]. Moreover, it is worth noting that the charge threshold voltage must not be confused with the extrapolated threshold voltage $V_{\text {text }}$ of the linear region of $I_{d}\left(V_{g}\right)$ to zero drain current. Indeed, when the mobility reduction factor $\theta$ is not equal to zero, $v_{\text {text }}$ is smaller than the charge threshold voltage $v_{t}$ [4]. $v_{\text {text }}$ is strictly equal to $v_{t}$ only when the mobility is gate voltage independent.

The mobility reduction factor $\theta$ can be determined by the following expression, which is constant in strong inversion :

$\theta=\left[I_{d} /\left(g_{m} v_{d}\left(v_{g}-v_{t}\right)\right)-1\right] /\left(v_{g}-v_{t}\right)$.

\section{b) Experiment}

Measurements have been carried out on n-channel MOSFETs fabricated at the LETI/LIR with a technology adapted for low temperature operation, with a surface channel doping of $1.5 \times 1015 \mathrm{~cm}^{-3}$, a gate oxide thickness of $120 \mathrm{~nm}$, and different geometries. Therefore, the threshold voltages are very low at ambient temperature (around $\mathrm{OV}$ ) and become higher at low temperature. Fig. 1 (a) and (b) show typical plots of the drain current $I_{d}\left(V_{g}\right)$ and the field effect mobility $\mathrm{MFE}\left(\mathrm{V}_{\mathrm{g}}\right)$ (which is obtained from the normalized transconductance by : MFE $=g_{m} /\left((Z / L) \cdot C_{o x}\right)$. Note the sub-linear dependence of $I_{d}$ above threshold due to the decrease of the mobility at high gate voltages. Fig. 1 (c) presents the corresponding plot of the $I_{d} /\left(g_{m}\right)^{1 / 2}$ characteristics. As expected, a linear dependence with gate voltage is obtained. The previous method allows the determination of the ambient temperature MOSFET parameters : $V_{t}=-0.035 \mathrm{~V}, \mu_{0}=1236 \mathrm{~cm}^{2} / \mathrm{N} . \mathrm{s}$, and $\theta=0.039 \mathrm{~V}-1$. Moreover, we can observe that $V_{t}$ is higher than the extrapolated threshold voltage $\left(V_{\text {text }}=-0.065 \mathrm{~V}\right)$, and $\mu_{0}$ is greater than the maximum field effect mobility (MFEmax $=1214 \mathrm{~cm}^{2} / \mathrm{V} . \mathrm{s}$ ). This method has also been successfully applied at liquid nitrogen temperature, demonstrating the validity of the previous relations for this temperature range.

\section{3 - EXTRACTION METHOD AT LIQUID HELIUM TEMPERATURE}

a) Theory

At very low temperature (4-40K), the conventional relations are no longer valid, and thus the previous extraction method cannot be applied. Indeed, the effective mobility 
of an inversion layer at very low temperature has a bell-shaped behaviour [5] with inversion charge $Q_{i}\left(M_{\text {eff }}=2 M_{m} /\left(Q_{j} / Q_{m}+Q_{m} / Q_{j}\right)\right.$, where $M_{m}$ is the maximum mobility and $Q_{m}$ is the corresponding inversion charge). Therefore, the effective mobility in strong inversion can be expressed by using the classical relation of the inversion charge $\left(Q_{j}=\right.$ $\left.C_{o x}\left(V_{g}-V_{t}\right)\right)$ as :

Meff $=2 \mu_{m} \theta^{\prime}\left(V_{g}-V_{t}\right) /\left[1+\theta^{\prime z}\left(V_{g}-V_{t}\right)^{z}\right]$, where $\theta^{\prime}=C_{0 x} / Q_{m}$ is the mobility parameter which characterizes the gate voltage dependence of Meff at low temperature.

As for ambient temperature, the basic idea is to eliminate the effect of the effective mobility dependence on gate voltage. This is achieved by using the following function, obtained by combining the drain current and normalized transconductance which are expressed using the low temperature mobility law :

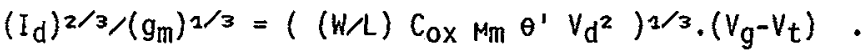

This quantity provides both the charge threshold voltage $V_{t}$ and the maximum mobility $M_{m}$, since $\theta^{\prime}$ can be determined from the maximum of $g_{m}$ given by :

$v_{\text {gmax }}=v_{t}+1 /\left(\sqrt{3} \theta^{\prime}\right)$

b) Experiment

Fig. 2 shows the transfer characteristics, the field effect mobility and the $I_{d^{2 / 3} / g^{1 / 3}}$ function. We can observe the expected linear dependence of this function on the gate voltage which confirms the validity of the model used in the parameter extraction. The previous method enables the determination of the low temperature MOSFET parameters $: V_{t}=$ $0.171 \mathrm{~V}, \theta^{\prime}=0.115 \mathrm{~V}^{-1}$, and $\mu_{m}=4589 \mathrm{~cm}^{2} / \mathrm{V} . \mathrm{s}$. Two interesting points stand out $: V_{t}$ is lower than the extrapolated threshold voltage $\left(V_{\text {text }}=1.78 \mathrm{~V}\right)$, and $\mathrm{Mm}_{\mathrm{m}}$ is lower than the maximum field effect mobility (MFEmax $=5600 \mathrm{~cm}^{2} / \mathrm{V} . \mathrm{s}$ ), which is not the case for ambient temperature $\left(V_{t} \geqslant V_{\text {text }}\right.$ and $M_{0} \geqslant$ MFEmax $)$.

\section{4 - CONCLUSION}

An original method of MOSFET parameter extraction has been proposed to avoid the effects of mobility reduction with gate voltage on the determination of both the threshold voltage and the mobility parameters. Different methods have been successfully tested for ambient, nitrogen and helium temperature ranges.

\section{REFERENCES}

[1] De la MONEDA F.H. et a1, IEEE Electron Devices Letters, EDL-3, p.10 (1982)

[2] CHU HAO et al, Solid-State Electronics, 28, p. 1025 (1985)

[3] KRUTSICK T.J. et al, IEEE Trans. Electron Devices, ED 34, p. 1676 (1987)

[4] GHIBAUDO G., Phys. Status Solidi (a), 95, p. 323 (1986)

[5] GHIBAUDO G. and F. BALESTRA, Solid-State Electronics, 31, p. 105 (1988) 

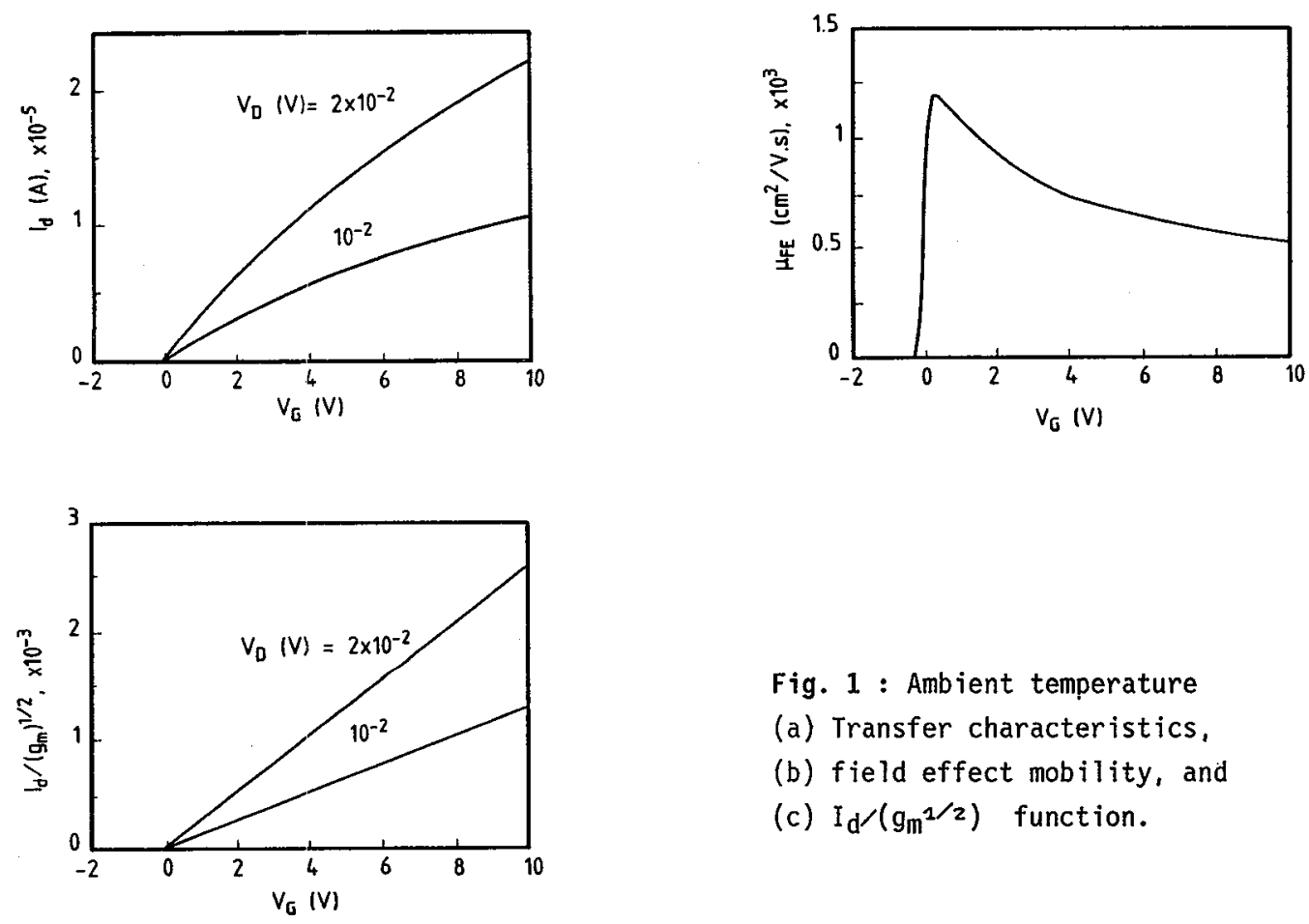

Fig. 1 : Ambient temperature

(a) Transfer characteristics,

(b) field effect mobility, and

(c) $I_{d} /\left(g_{m^{1 / 2}}\right)$ function.
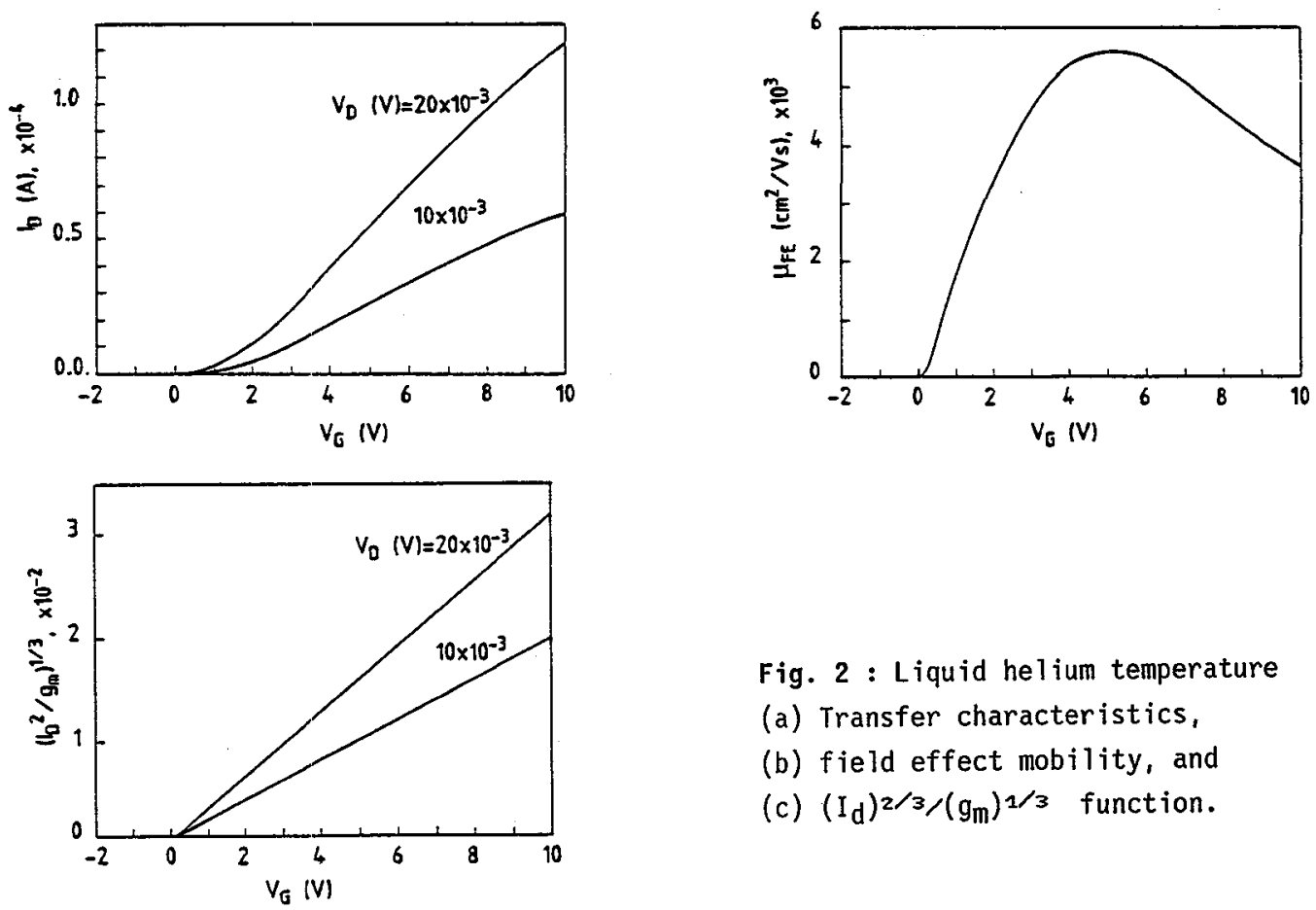

Fig. 2 : Liquid helium temperature

(a) Transfer characteristics,

(b) field effect mobility, and

(c) $\left(I_{d}\right)^{2 / 3} /\left(g_{m}\right)^{1 / 3}$ function. 